\title{
Dispersive kinetics in discotic liquid crystals
}

\author{
O. Kruglova* \\ Laboratoire Interfaces \& Fluides Complexes, Centre d'Innovation et de Recherche en Matériaux Polymères, Université de Mons Hainaut, \\ 20, Place du Parc, B-7000 Mons, Belgium \\ F. M. Mulder \\ Department of Radiation, Radionuclides \& Reactors, Faculty of Applied Science, Delft University of Technology, Mekelweg 15, \\ 2629 JB Delft, The Netherlands \\ G. J. Kearley \\ Bragg Institute, Building 87, Australian Nuclear Science and Technology Organisation, PMB 1 Menai, New South Wales 2234, Australia
}

S. J. Picken

Polymer Materials and Engineering, Department of Material Science and Technology, Julianalaan 136, 2628 BL Delft, The Netherlands

J. A. Stride

Institute Laue Langevin, BP 156, Grenoble 38042 Cedex 09, France and School of Chemistry, University of New South Wales, Sydney, New South Wales 2052, Australia

I. Paraschiv and H. Zuilhof

Laboratory of Organic Chemistry, Dreijenplein 8, 6703 HB Wageningen, The Netherlands

(Received 8 April 2010; revised manuscript received 15 October 2010; published 22 November 2010)

\begin{abstract}
The dynamics of the discotic liquid-crystalline system, hexakis ( $n$-hexyloxy) triphenylene (HAT6), is considered in the frame of the phenomenological model for rate processes proposed by Berlin. It describes the evolution of the system in the presence of the long-time scale correlations in the system, and we compare this with experimental quasielastic neutron scattering of the molecular assembly of HAT6 in the columnar phase. We interpret the parameters of this model in terms of nonextensive thermodynamics in which rare events in the local fast dynamics of some parts of the system control the slower dynamics of the larger molecular entity and lead to a fractional diffusion equation. The importance of these rare local events to the overall dynamics of the system is linked to the entropic index, this being obtained from the data within the model approach. Analysis of the waiting-time dependence from momentum transfer reveals a Lévy distribution of jump lengths, which allows us to construct the van Hove correlation function for discotic liquid-crystalline system.
\end{abstract}

DOI: 10.1103/PhysRevE.82.051703

PACS number(s): 61.30.-v, 78.70.Nx, 05.45.-a

\section{INTRODUCTION}

Discotic liquid-crystalline systems consist of disklike molecules that self-assemble into columns as a result of overlap of the $\pi$ orbitals of the aromatic cores $[1,2]$. Electrons and holes hop along the column direction and that has drawn attention to these materials for nanoscale conductive devices. Since the interdisk hopping occurs on the picosecond time scale [1] and the lifetime of charge carriers is on a nanosecond time scale [3], we anticipate that the dynamics within these time scales will be particularly relevant for transient conductivity. The transient conductivity is sensitive to the dynamics of the cores, but the alkoxy tails not only assist the self-assembly (by phase separation of aromatic and aliphatic parts) but also play a crucial role in the dynamics of the cores. In our study, preference has been given to hexakis (n-hexyloxy) triphenylene (HAT6) as it brings together enough simplicity to allow a rather complete interpretation of data yet contains the basic structural entities that allow our

\footnotetext{
*o.v.kruglova@gmail.com
}

study to be extended to other complex systems that have better electronic properties. There has been considerable recent study on larger systems such as hexabenzocoronene using NMR and atomistic modeling methods [4-8], which demonstrates the importance of this type of material.

We choose to develop our model from the experimental technique, quasielastic neutron scattering (QENS), because this technique simultaneously follows hydrogen-atom dynamics over picosecond time scales and length scales of angstroms. van Hove [9] established that the neutron-scattering function $S(Q, \omega)$, where $Q$ is the momentum transfer and $\omega$ is the energy transfer which is measured in a QENS experiment, represents the Fourier transforms over $\boldsymbol{r}$ and $t$ of the generalized pair distribution function in space and time $G(\boldsymbol{r}, t)$. In general, it describes the correlation between the presence of the particle at the position $\boldsymbol{r}^{\prime}+\boldsymbol{r}$ at time $t^{\prime}+t$ and the presence of the particle in position $\boldsymbol{r}^{\prime}$ at time $t^{\prime}$. Under certain conditions this can be simplified and represents an average density for homogeneous statistically independent systems. Such an approximation allows classical models for $G(\boldsymbol{r}, t)$ to be used and to represent $S(Q, \omega)$ as a sum of elastic and quasielastic parts [10]. This assumption is no longer valid for complicated systems [11] in which interactions 


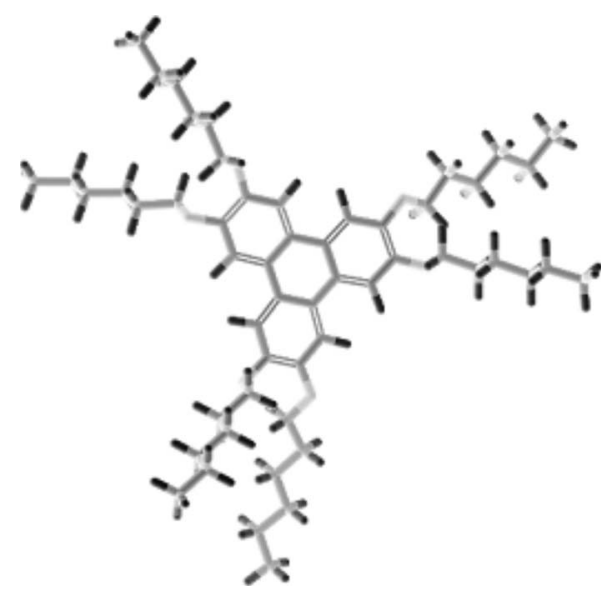

FIG. 1. Structure of HAT6.

within the system must be taken into account. Even initial fitting of the QENS data of the discotic liquid-crystal HAT6 reveals "longer than" exponential relaxation and this is one of many examples of complicated dynamics where a conventional, simplified, approach fails. There are two possible approaches to the analysis of data of this type. First, we may fit the so-called stretched exponential and try to relate the variation of the parameters of this analytical function ( $\beta$ and $\tau$ will be defined later) to some characteristics of the system. Alternatively — and preferably_we can consider the molecular structure and the columnar assembly of HAT6 and try to characterize their dynamics with a physically meaningful model that agrees with the experimental QENS data. It has been established many times that such models produce a relaxation curve that is virtually indistinguishable from a stretched exponential unless measurements are made over many orders of magnitude in time. The advantage of our approach is that we can extract the variation of the physically meaningful parameters as a function of temperature and momentum transfer $(Q)$ from the experimental data and thus gain some new insight into the dynamics of the system. If our model is incorrect then it will only agree with experimental data, both temperature and $Q$ dependence, by using physically unreasonable parameters. Using a simple stretched exponential any insight gained from the variation of $\beta$ and $\tau$ will be tenuous.

In this paper we analyze our data with a model for the kinetics of a system that is coupled to a relaxing environment, taking into account the interactions between different parts of the system and correlation effects. When combined with a fractional diffusion equation this allows us to construct the space-time correlation function for the system, $G(\boldsymbol{r}, t)$, which we compare directly with the Fourier transform of the experimentally measured function.

\section{EXPERIMENTAL TECHNIQUE}

QENS spectra for HAT6 (Fig. 1) were obtained using the cold neutron time-focussing time-of-flight spectrometer (IN6) spectrometer at Institute Laue Langevin in France with an incident wavelength of $5.9 \AA$. Measurements were per- formed at temperatures of $T=340,352,358,364$, and $370 \mathrm{~K}$ for the protonated samples and at $T=367 \mathrm{~K}$ for the taildeuterated analog, temperature control being achieved with a standard heating loop. Formally, $340 \mathrm{~K}$ corresponds to the crystalline $\rightarrow$ liquid crystalline phase transition and $T=370 \mathrm{~K}$ to the liquid crystalline $\rightarrow$ liquid phase transition,

$$
\text { crystalline } \stackrel{340 \mathrm{~K}}{\rightarrow} \text { liquid crystalline } \stackrel{370 \mathrm{~K}}{\rightarrow} \text { liguid. }
$$

Our interest is solely in the columnar liquid-crystal phase in which molecules within a column are held together by $\pi-\pi$ orbitals overlap of the aromatic cores and the van der Waals interactions of the "phase-separated" alkyl tails. In the crystalline phase where the molecules are arranged in a herringbone structure there is no charge transfer and the intermolecular coupling of dynamics would in any case have a different relevance to the electronic properties of the material. We, therefore, restrict our study to the columnar phase.

Data were corrected using standard algorithms and analyzed using locally written routines. We have extended our previous measurements on this system by using samples in which the alkoxy tails are deuterated [12] in order to highlight the dynamics of the aromatic disk cores. The purity of HAT6 and HAT6 with deuterated tails was verified with mass spectrometry.

\section{MODEL}

Studies of the dynamics of complex systems such as polymers, biological molecules, or liquid crystals have revealed deviations from the behavior described by diffusion equations and represented by a Gaussian function [13-15]. There has been considerable effort to find models for the dynamical behavior that lead to anomalous diffusion [16,17], but many of these lack a mathematical description of the processes that provides the equation of motion with which of the behavior of complex systems of known initial conditions could be predicted. Although we attempted to analyze our data using existing homogeneous (each process relaxes nonexponentially) and heterogeneous (relaxation processes are exponential but with relaxation-time distribution) models, these are unable to describe the $Q$ dependence of our experimental data. For this, data were fitted with stretched exponential function to determine the dependence $\tau^{\beta}$ on $Q$, as illustrated in Fig. 2. In the heterogeneous case $\tau^{\beta} \propto Q^{-2}$, while in homogeneous case it should be $\tau^{\beta} \propto Q^{-2}$ with $\beta=1$. It is clear from Fig. 2 that neither of these scenarios agrees with our experimental data and for that, therefore, we need alternative model to describe behavior of discotic liquid crystals.

A natural generalization of the diffusion equation is represented by a fractional diffusion equation as suggested by Metzler and Klafter [18],

$$
\frac{\partial W(r, t)}{\partial t}={ }_{0} D_{t}^{1-\beta} \frac{\sigma^{\alpha}}{\tau^{\beta}} \nabla^{\alpha} W(r, t),
$$

where $W(r, t)$ is the probability distribution function, $\alpha$ and $\beta$ are the characteristic space and time exponents, respectively, ${ }_{0} D_{t}^{1-\beta}$ is the Riemann-Liouville fractional operator, $\sigma$ is characteristic jump length, and $\tau$ is a characteristic waiting time. 


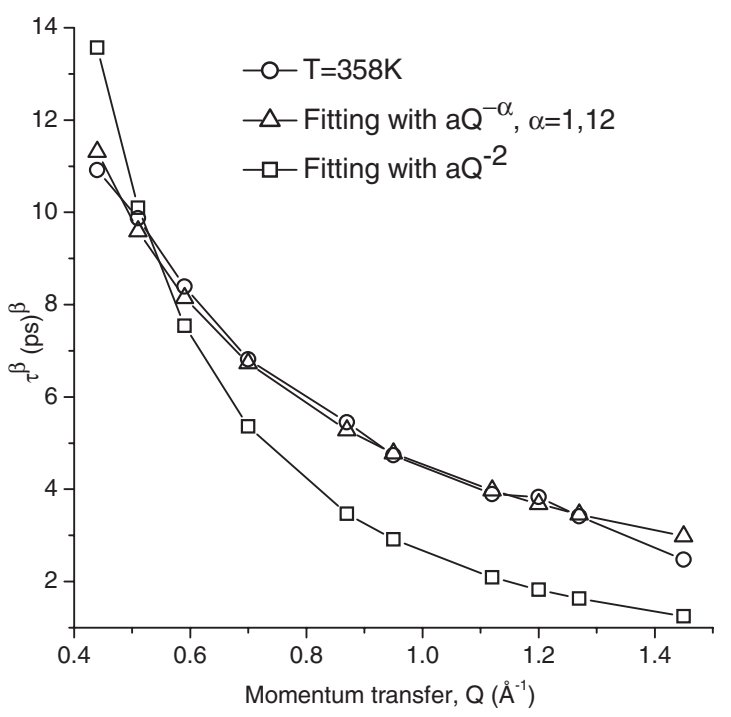

FIG. 2. Dependence of waiting times exponentiated in the power $\beta$ obtained from fitting intermediate scattering function with a stretched exponential dependence $S_{\text {self }}(Q, t)=A(Q) \exp \left[-(t / \tau)^{\beta}\right]$. This was fitted with $\tau^{\beta} \propto Q^{-2}$, open squares, and with $\tau^{\beta} \propto Q^{-\alpha}$, open triangles, with $0,41<\beta<0,48$. The waiting-time dependence is in better agreement with $\tau^{\beta} \propto Q^{-\alpha}$ dependence.

Under the conditions $\alpha=2$ and $\beta=1$, the fractional diffusion equation transforms into Fick's second law and the solution then appears in terms of the familiar Gaussian function (see Ref. [18] for details). If the waiting-time probability distribution function is broad, then the time relaxation will be described by the Mittag-Leffler function, $E_{\alpha}\left(-(t / \tau)^{\alpha}\right)$ $=\sum_{n=0}^{\infty}\left(-(t / \tau)^{\beta}\right)^{n} / \Gamma(1+\beta n)$, instead of an exponential function. But despite its obvious advantages, the fractional diffusion equation does not give any detailed description of the anomalous diffusion or a representation of the time exponent and waiting time. Our aim is to reveal the fundamental mechanism, and for this we will consider the evolution of the time part of the fractional diffusion equation. We will adopt one of the simplest phenomenological models for rate processes that have been proposed by Berlin et al. [19], since intuitively this would seem appropriate to our system. This model is based on the configurational-energy approach for the system with a static disorder, which suggests the presence of more than one configuration energy distribution, acting as traps with depth $E_{i}$. Such an assumption is especially justified for complex materials where an extensive variation of interaction energies is possible and, therefore, there will be an extensive variation not only of activation energies but also of traps. The characteristic time of remaining in a trap, $\tau$, will be determined by

$$
\tau_{i}=\frac{1}{Z w_{0}} \exp \left(\frac{E_{i}}{k_{B} T}\right),
$$

where $Z$ is the number of states different from $i$ th state, $w_{0}$ is the jump frequency of normal diffusion, $k_{B}$ is the Boltzmann constant, and $T$ is the temperature. Any excitation of such media, for example, slight change of temperature or pressure, causes a rearrangement of configurational states and relax- ation toward energy equilibrium. In complex systems such as polymers, liquid crystals, etc., relaxation rates toward lower energies for different pairs of trapping sites will vary over a very wide range,

$$
k_{i}=k_{0} \exp \left(-\frac{\eta E_{i}}{k_{B} T}\right),
$$

where $k_{0}$ is pre-exponential parameter and $\eta$ is a parameter whose physical meaning will be discussed further. Clearly, states (or degrees of freedom) that require less energy to move toward equilibrium will relax first, and hence hierarchical order in relaxation is present in such a system. This leads to the familiar delayed response in comparison to a system in which there is little interaction between its parts (hierarchical order is absent) and relaxation occurs in a parallel way.

This type of evolution can be understood in terms of a survival probability of the initial state $P(t)=\Sigma_{i} p_{i}(t)$, where $p_{i}(t)$ is the probability of the $i$ th state to be populated at time $t[19]$ and is represented by a Kolmogorov-Feller integral equation, which is suitable for solving the kinetic problems,

$$
\frac{d p_{i}(t)}{d t}=-k_{i} p_{i}(t)+\sum_{i, j}\left[w_{j i} p_{j}(t)-w_{i j} p_{i}(t)\right] .
$$

We will show later that $P(t)$ is equivalent to the Fourier transform of the function measured in the QENS experiment. By assuming uncorrelated transitions and introducing a Laplace transform, a general solution is found in terms of the initial population distribution $G(E)$ and the density of substates $g(E)$.

Let us consider one particular case, $T<T_{0}$, which is the most applicable to our system, where $T$ is the temperature and $T_{0}$ is the temperature at which the system would have Gaussian behavior (where $T_{0}$ is related to the parameter $E_{0}$ of the exponential distribution of substates),

$$
G(E)= \begin{cases}\frac{1}{E_{0}} \exp \left(-\frac{E}{E_{0}}\right) & \text { for } E \geq 0 \\ 0 & \text { for } E \leq 0 .\end{cases}
$$

Under these conditions the general solution can be reduced to following expression:

$$
P(t)=E_{\beta}\left(-(t / \tau)^{\beta}\right)=\sum_{k=0}^{\infty} \frac{(-1)^{k}(t / \tau)^{\beta k}}{\Gamma(1+\beta k)},
$$

where $\tau$ is the waiting time that is defined as

$$
\tau=\frac{1}{Z w_{0}}\left[\frac{\pi Z w_{0} \beta}{k_{0} \sin (\pi \beta)}\left(1+(\eta-1) \frac{1}{\beta}\right)\right]^{1 / \beta}
$$

and $\beta$ is the dispersion parameter defined as $\beta=T / T_{0}$ and $0<\beta<1$. The solution of this kinetic equation is identical to that of the fractional diffusion equation with a broad waitingtime distribution. We propose that our model is appropriate to the discotic system in which $\pi$ - $\pi$ interactions connect the triphenylene cores, and there is entanglement and interdigitation of the alkoxy tails between neighboring disks and columns. The kinetic equation would then also be appropriate. 
Since the solution was obtained with the condition of an exponential distribution of substates, one would expect to obtain the same type of distribution for noncorrelated jumps and a different type for correlated jumps after a characteristic waiting time. For that purpose let us analyze expression (6). The expression for the diffusion waiting time differs from that of normal diffusion and clearly will not have a simple exponential form. Transforming it with respect to $Z$ we obtain the following representation:

$$
Z=\left[\frac{\pi \beta}{\tau^{\beta} w_{0}^{\beta-1} k_{0} \sin (\pi \beta)}\right]^{1 / \beta-1}\left(1+(\eta-1) \frac{1}{\beta}\right)^{1 / \beta-1} .
$$

Assuming

$$
C=\left[\frac{\pi \beta}{k_{0} \tau^{\beta} w_{0}^{\beta-1} \sin (\pi \beta)}\right]^{1 / \beta-1}
$$

as a normalization constant, we obtain the expression for the substate distribution,

$$
Z=C\left(1+(\eta-1) \frac{1}{\beta}\right)^{1 / \beta-1} .
$$

One can immediately see that expressions (4) and (9) are different. After a characteristic waiting time, the function type of the initial distribution of substates has changed. Comparing Eq. (9) and the Tsallis distribution, $p(x) \propto[1-(1-q) x]^{1 / 1-q}$, we can see close similarities, the important quantity here being the entropic index $q$ [20]. But we can also obtain such a distribution by other means [21].

Let us consider a small volume in our system. The average number of energy traps in this volume is defined as $\left\langle n_{i}\right\rangle=E_{i} / E_{0}$ and the probability distribution of encountering $n_{i}$ in this volume is Poissonian [22],

$$
P\left(E_{i}\right)=\frac{\left\langle n_{i}\right\rangle^{n} e^{-\left\langle n_{i}\right\rangle}}{n !} .
$$

Assuming that some number of subsystems is trapped in this volume, then the probability of finding $\zeta$ subsystems in those traps is given by a gamma distribution,

$$
f_{\zeta}\left(E_{i}\right)=\frac{1}{E_{0} \Gamma(\zeta)}\left(\frac{E_{i}}{E_{0}}\right)^{\zeta-1} \exp \left(-\frac{E_{i}}{E_{0}}\right) .
$$

We can now find the expectation value of the number of subsystems $Z$ that are distinct from those associated with the $i$ th trapping sites, taking into account that some of them are still trapped and some are relaxing out of the $i$ th trapping sites,

$$
\begin{aligned}
Z & =\int_{0}^{\infty} Z \tau_{i} k_{i} f_{\zeta}\left(E_{i}\right) d E_{i} \\
& =\frac{k_{0}}{w_{0} \Gamma(\zeta)} \int_{0}^{\infty} \exp \left\{-\xi\left[1+(\eta-1) \frac{E_{0}}{k_{B} T}\right]\right\}(\xi)^{\zeta-1} d \xi
\end{aligned}
$$

where $\xi=E_{i} / E_{0}$. If we substitute $t=\xi\left[1+(\eta-1)\left(E_{0} / k_{B} T\right)\right]$ then the integral will be expressed as

$$
\begin{aligned}
Z & =\frac{k_{0}}{w_{0} \Gamma(\zeta)}\left(1+(\eta-1) \frac{T_{0}}{T}\right)^{-\zeta} \int_{0}^{\infty} e^{-t} t^{\zeta-1} d t \\
& =\frac{k_{0}}{w_{0}}\left(1+(\eta-1) \frac{T_{0}}{T}\right)^{-\zeta} .
\end{aligned}
$$

If we define

$$
\zeta=\frac{1}{q-1}
$$

then

$$
Z=\frac{k_{0}}{w_{0}}\left[1+(\eta-1) \frac{T_{0}}{T}\right]^{1 / 1-q} .
$$

The expression in curved brackets of Eqs. (9) and (11) is identical to the Tsallis distribution [20] even though Eq. (11) is not normalized. We will thus equate $\eta$ with $q$ and so make the connection to the experimentally determined value of $\beta(0<\beta<1)$,

$$
q=\eta=2-\beta .
$$

Expressions (10) and (12) connect the entropic index $q$ with number of trapped subsystems and the equilibrium temperature $T_{0}$ since $\beta$ is defined by means of $T_{0}$. The closer the temperature is to $T_{0}$, the more parts of the system are trapped in particular states that correspond to equilibrium. In this case $q \rightarrow 1$. The opposite case is when the temperature is far from the equilibrium value. Then just one part of system will be in a particular trap. This case represents $q \rightarrow 2$. Therefore, we conclude that the entropic index will fall in $1<q<2$.

It is well established that the van Hove neutron-scattering function $S(Q, \omega)$ represents the double Fourier transform of the generalized pair distribution function $G(r, t)$ [9]. The latter is traditionally analyzed via a diffusion equation and kinetic equation of the Chudley-Elliot model [23] in terms of the Gaussian function. Equation (3) can be easily transformed into the equation used in the Chudley-Elliot model. One of the assumptions made there is that there is negligible time for the jump from one site to another. That time is described in Eq. (3) by the first term on the right side of the equation, relaxation rate $k_{i}$. Another assumption is that time between two jumps and the distance between two sites are the same for all sites. With these assumptions, the ChudleyElliot kinetic equation is obtained from Eq. (3), which generalizes the Chudley-Elliot model. It has been shown that solution of the Chudley-Elliot model equation is the intermediate neutron-scattering function [24] and thus the solution of Eq. (3) is also the intermediate neutron-scattering function. It follows that the probability of remaining in the initial configuration, $P(t)$, is equivalent to the normalized intermediate function $F(Q, t)$, this being the Fourier transform of $S(Q, \omega)$ for IN6.

\section{RESULTS AND DISCUSSION}

As mentioned in Sec. I, a conventional approach and treatment of our data fails when dealing with such complicated system as liquid crystals, and fitting with two Lorent- 


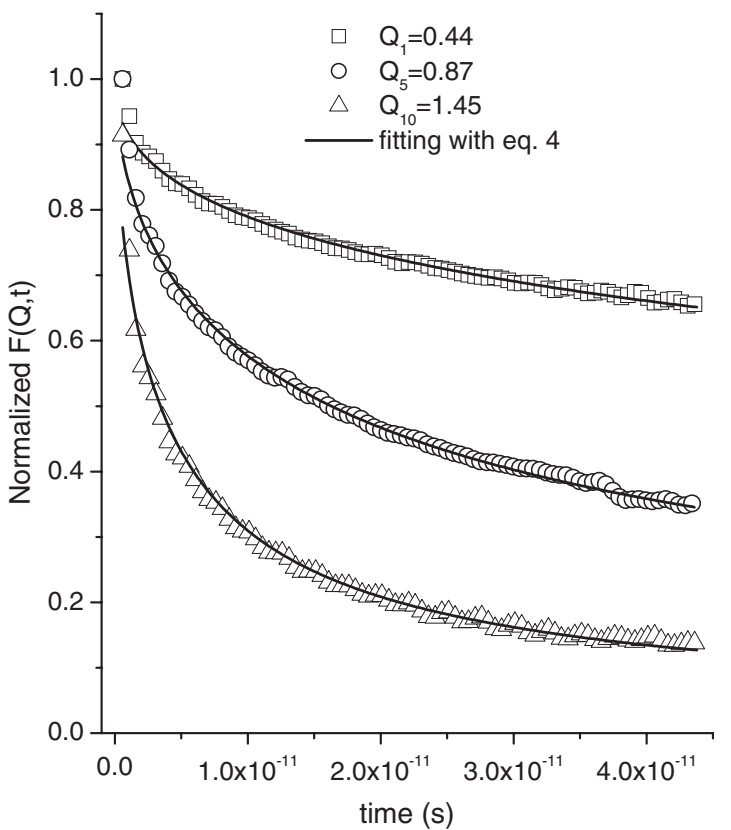

FIG. 3. Intermediate scattering function for HAT6 at $T=358 \mathrm{~K}$ for $Q=0.44,0.87$, and $1.45 \AA^{-1}$ fitted with Eq. (3).

zians has systematic deviation from the shape of the experimental curve. Of course, fitting with three or even more the Lorentzian functions would give better fit although the physical meaning of such an introduction will be ambiguous. The liquidlike contribution from the alkoxy tails is removed in the sample with deuterated tails, and spectra from this sample show an even greater deviation from the simple twoLorentzian quasielastic profile. In this case $S(Q, \omega)$ reflects mostly the response from the cores, which are ordered in a hexagonal lattice and, therefore, should follow the conventional diffusion equation. Therefore, this example becomes especially illustrative. For interpretation of our data we have used a different approach and model for the relaxation kinetics in a correlated environment. To analyze our data in the time domain, $S(Q, \omega)$ has to be converted using an inverse Fourier transform (IFT) and dividing by the IFT of the measured resolution function to obtain the pure response of the system. In this way we do not separate the elastic and quasielastic parts of the signal but study the evolution of the system as a whole one. This assumes that there is no independent "static" component that gives only elastic scattering (within the resolution limits). All intermediate scattering functions $F(Q, t)$ have nonexponential slopes (Fig. 3), this fact indicating that the motion of the molecules in this system is complex. Our interest here is in the time dependent entanglement of the alkoxy tails and how this affects the dynamics of the aromatic cores.

For the fitting procedure we have used 50 terms in the summation of Eq. (5) since a greater number were found not to change the overall fitting quality. Figure 3 illustrates the result of fitting the IFT of the QENS signal by Eq. (5) with only $\tau$ and $\beta$ as free parameters. Therefore, $\tau$ and $\beta$ may have different values for each value of $Q$. The behavior of HAT6 is described quite well by expression (5), which indicates that we are probing a single relaxation process of hy-

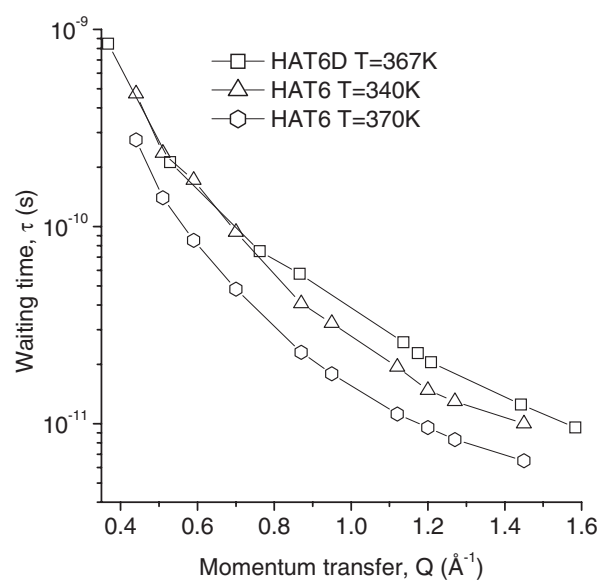

FIG. 4. Waiting time, $\tau$, dependence on momentum transfer, $Q$, for HAT6 with deuterated tails at $T=367 \mathrm{~K}$ and HAT6 with protonated tails at temperatures close to phase transition $T=340 \mathrm{~K} \quad$ (crystalline $\rightarrow$ liquid crystalline) and $T=370 \mathrm{~K}$ (liquid crystalline $\rightarrow$ isotropic).

drogen atoms, reflecting a system of collective motion over the entire data.

It is crucially important to examine the value of $\tau$ as a function of momentum transfer $Q$ (Figs. 4 and 5) because this reflects changes in the waiting time as a function of distance. Figure 3 represents the $Q$ dependence of $\tau$ at $T=340$ and $370 \mathrm{~K}$ for the protonated sample (phasetransition temperatures HAT6) and at $T=367 \mathrm{~K}$ (phasetransition temperature) for HAT6 with deuterated tails. The most striking feature of this figure is that the behavior of HAT6 with protonated tails at $340 \mathrm{~K}$ resembles that of the effective core motion coming from the sample with deuterated tails at $367 \mathrm{~K}$ until $Q \sim 0.66 \AA^{-1}$, corresponding to a diameter of $\sim 9 \AA$. This is significantly larger than a static core and probably reflects the sliding motion of the cores over each other and the participation of the $\mathrm{O}$ atom plus the first one-or-two $\mathrm{CH}_{2}$ units as part of the effective core [25]. At length scales shorter than this, we see the fast local relaxation of the chains in the protonated tail sample, and at

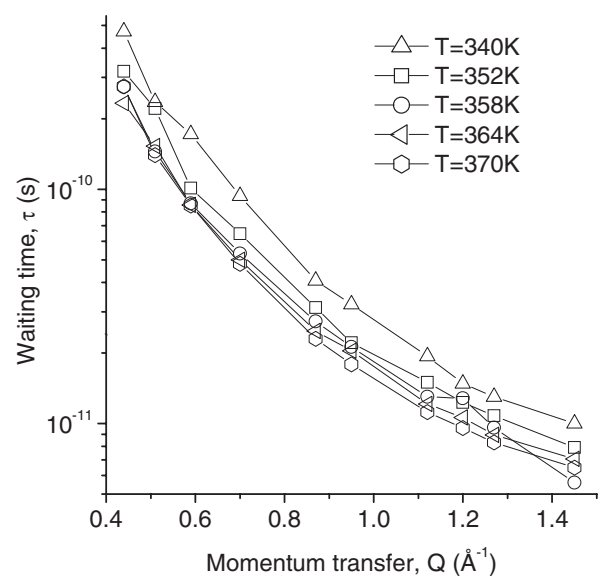

FIG. 5. Waiting time, $\tau$, dependence on momentum transfer, $Q$, for different temperatures $T=340,352,358,364$, and $370 \mathrm{~K}$, protonated HAT6. 


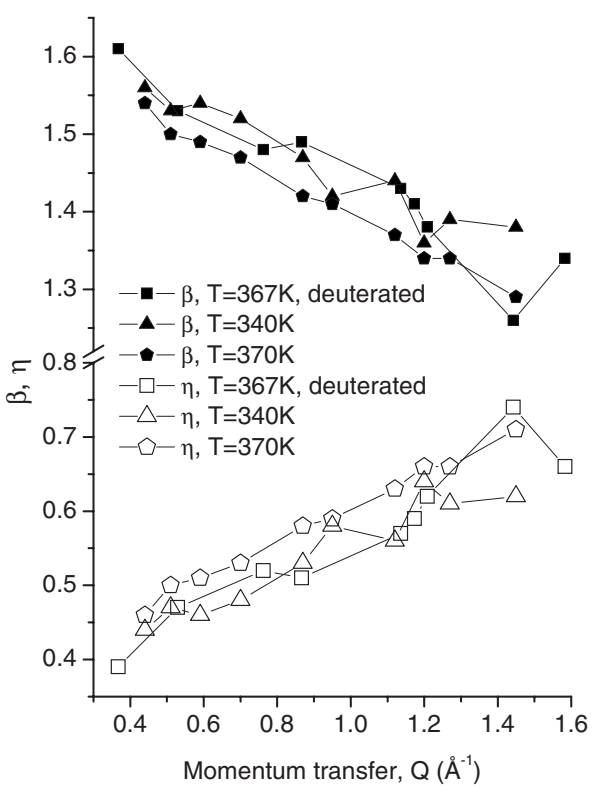

FIG. 6. Dispersion parameter $\beta$ and entropic index, $\eta$, dependences on momentum transfer, $Q$, for HAT6 with deuterated tails at $T=367 \mathrm{~K}$ and HAT6 at temperatures close to the phase transitions $T=340 \mathrm{~K} \quad$ (crystalline $\rightarrow$ liquid crystalline) and $T=370 \mathrm{~K}$ (liquid crystalline $\rightarrow$ isotropic).

longer scales we see more the effect of the core dragging on and confining the entangled tails. Comparing that with $\tau(Q)$ at $370 \mathrm{~K}$ reveals that the waiting time becomes significantly shorter and it is possible that motion of the tails, visible in the protonated sample, plays a determinative role. The core is still slower as can be seen from the deuterated tail sample at roughly the same temperature that confirms findings of Dvinskikh et al. [26]. It is interesting to see how the waiting time changes in temperature range $340<T<370 \mathrm{~K}$ (Fig. 4) where the system is in the liquid-crystalline phase. We see in Fig. 5 that the value of the waiting time for a protonated sample decreases as the temperature increases from which we concludes that tails become less entangled, relaxing toward equilibrium more quickly, as has already been observed in the similar system hexapentyloxytriphenylene (HAT5) [27]. In the region, $Q>0.6 \AA^{-1}$, values of the waiting time are almost the same, except for $T=352 \mathrm{~K}$, and they tend to diverge for $Q<0.6 \AA^{-1}$. It is likely that at $T=352 \mathrm{~K}$ the effect of the core motion in slowing down the tail motion is still pronounced although it is reduced by the tails. The dynamics in the discotic core-tail system are clearly correlated, the detailed dynamics of the tails not being simply additive to that of the cores.

Figures 6 represents change in dispersion parameter $\beta$ with momentum transfer $Q$. The dispersion parameter increases gradually with increasing $Q$, but it is always less than 1 , which indicates that motion in HAT6 takes place in subdiffusive regime [18]. Again, values $\beta(Q)$ for samples with deuterated tails and a protonated one at $T=340 \mathrm{~K}$ are very close to each other for $Q<0.66 \AA^{-1}$, which implies the importance of the core motion on this length scale at low temperatures. The tail motion plays a crucial role at temperatures close liquid crystalline $\rightarrow$ isotropic phase transition and, of

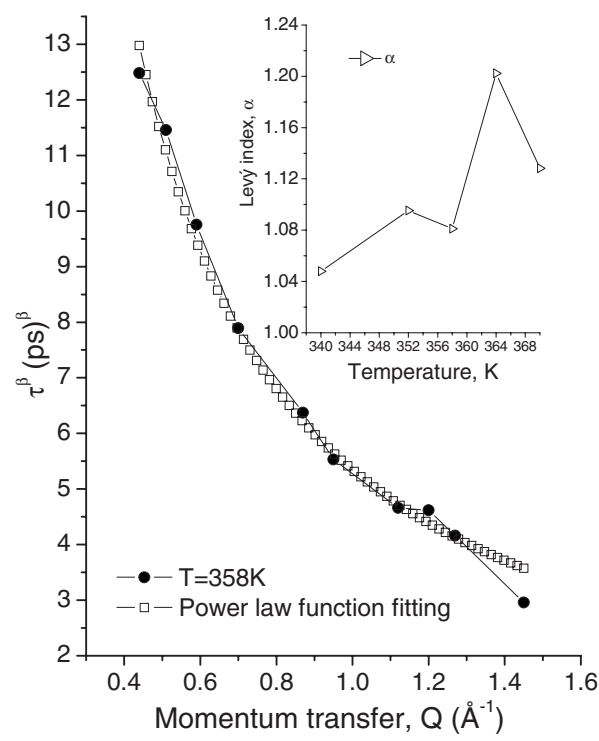

FIG. 7. Fitting of $\tau(Q)$ at $T=358 \mathrm{~K}$ with the power-law function $\tau^{\beta}=a Q^{-\alpha}$. Inset: temperature dependence of the Lévy index, $\alpha$, represents slow increase toward $\alpha=2$.

course, at short length scales. The relaxation in these discotic liquid crystals occurs in the subdiffusive regime $(0<\beta<1)$ [18] (Fig. 6) with a Tsallis distribution of states after characteristic waiting time that has the entropic index $\eta=q>1$ indicating a partially equilibrated system [21]. The Tsallis distribution of states indicates also the presence of long-range correlations in our system as well as hierarchical order [28]. With relation to discotic liquid crystals hierarchical order means a certain order of relaxation: states which need the minimum possible energy for escaping from trap to equilibrium, for example, stretching of $\mathrm{CH}$ groups, will relax first, then states which need more time and space, for example, methyl-group rotation, then translational motion of one of the tails, and so on.

The Brownian diffusion is characterized by a diffusion coefficient that is defined as $K=(\Delta x)^{2} / \tau$ or in terms of momentum transfer $K=a / Q^{2} \tau$. Therefore, the dependence of waiting time vs momentum transfer will be an inverse quadratic relation. But in the case of discotic liquid crystals it fails to show such dependence. This means that the jumplength variance no longer has a Gaussian distribution. In the fractional diffusion equation the diffusion coefficient is defined as $K_{\alpha}^{\beta} \equiv(\Delta x)^{\alpha} / \tau^{\beta}$ [18]. For this reason we have fitted that dependence with the power-law function $\tau^{\beta}=a Q^{-\alpha}$ (Fig. 7 ), the agreement being rather good. This result also agrees with dependence obtained by Arbe et al. [17], assuming that in their case $\alpha$ is equal to 2. Therefore, the jump-length variance distribution represents a Lévy distribution [18]. From a physical point of view this implies a higher than usual probability of long jumps in the system. The inset of Fig. 7 represents the temperature dependence of the Lévy index. There is a tendency of $\alpha$ to increase toward 2 when $T$ increases, indicating that with increased temperature, the system gains enough energy to move toward equilibrium with fewer long jumps. A slightly different picture emerges from dependence of $\tau^{\beta}$ vs $Q$ (Fig. 8). The Lévy index for HAT6 with deuterated tails that represent cores is smaller than 1 , 


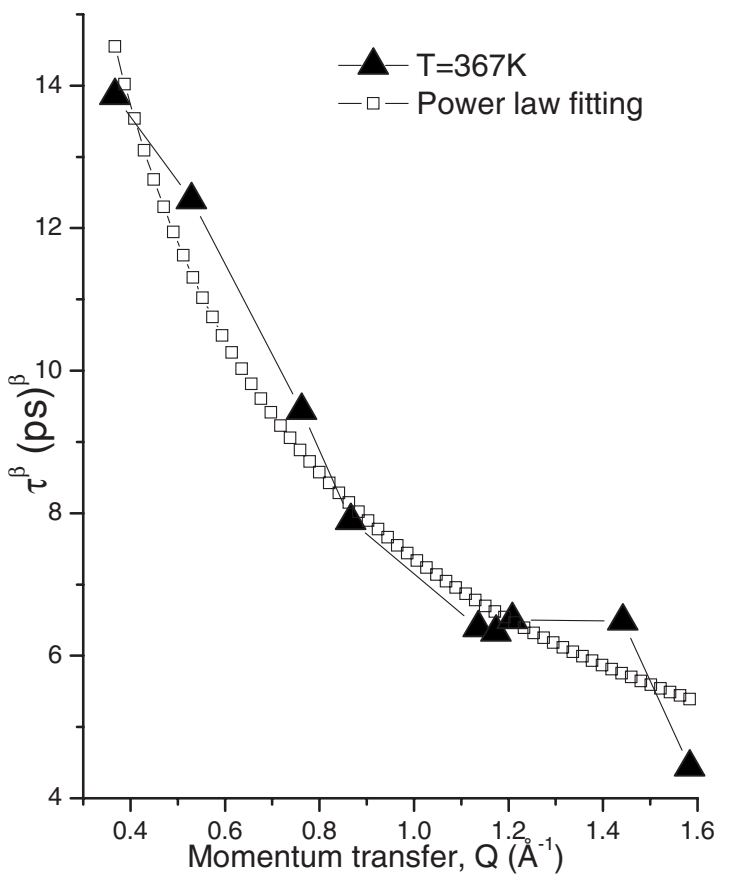

FIG. 8. Fitting of $\tau(Q)$ at $T=367 \mathrm{~K}$ with the power-law function $\tau^{\beta}=a Q^{-\alpha}$ for HAT6 with deuterated tails. The Lévy index is 0.68 that differs drastically from that of fully protonated HAT6 and reflects distribution of jumps length for triphenylene core.

0.68, suggesting that the core must experience several long jumps along with small jumps to reach an equilibrium state. Even though the temperature $T=367 \mathrm{~K}$ corresponds to the liquid crystalline to liquid transition temperature, the cores, unlike tails (Fig. 6 represents fully protonated HAT6 where response from tails dominates), seem to be more constrained in space. That implies the presence of some limited columnar order in HAT6 even at phase-transition temperature, in contrast to the tails that become less entangled with increasing temperature.

Knowing how waiting times and jump lengths are distributed and using the solution of the fractional diffusion equation [18], we can construct the van Hove correlation function from the general expression $S(\mathbf{Q}, s)=F_{r}\left\{L_{t}\{G(\mathbf{r}, t)\}\right\}=\{[1-w(s)] / s\}\{1 /[1-\psi(\mathbf{Q}, s)]\}[29]$, where $s=\sigma+i \omega$, complex frequency $w(s)$ is the transition probability, and $\psi(\mathbf{Q}, s)$ is the probability distribution function of making jump of reciprocal length $\mathbf{Q}$ in the frequency interval $s$ to $s+d s$. Fortunately, the Mittag-Leffler function, which corresponds to survival probability at initial state, has a direct representation in the frequency domain: $s^{-1}(\tau s)^{\beta} /\left[1+(\tau s)^{\beta}\right][19]$. The probability distribution func- tion of making jump $\psi(\mathbf{Q}, s)$ is decoupled for ordered arrays, as pointed out by Klafter et al. [29]. Since discotic liquid crystals can be treated as ordered arrays, we can express the probability distribution function as $\psi(Q, s)=w(s) \lambda(Q)$. Then it will be represented in terms of $s$ and $\mathbf{Q}$ as $\psi(\mathbf{Q}, s)=a Q^{\alpha} /\left[1+(\tau s)^{\beta}\right]$. Finally, the expression for the Laplace-Fourier space transform of the van Hove correlation function will be $S(Q, s)=s^{-1}(\tau s)^{\beta} /\left[1+(\tau s)^{\beta}-a Q^{\alpha}\right]$. Since the van Hove correlation function describes the behavior of the system in time and space, two characteristic exponents- $\beta$, which indicates on the presence of dispersed waiting times, and $\alpha$, which indicates on the presence of long jump lengths in system-define the behavior of the system. Therefore, in the case of discotics it will be represented by the nonMarkovian Lévy flight [18] with a broad distribution of waiting times and long jump lengths. So, the fractional space exponent $\alpha$ and the time exponent (or dispersion parameter) $\beta$ are fundamental characteristics of dynamics in liquidcrystalline system.

\section{CONCLUSION}

The present work demonstrates that the rather simple model for rate processes can be developed, which takes account of the structural aspects of the underlying molecular system and its assembly, and that this model agrees quite well with the quasielastic neutron-scattering data. In contrast to more general interpretations of subdiffusion using stretched exponentials, our model is developed by consideration of the system itself and hence provides meaningful parameters that have physically reasonable values. This enables us to understand the dynamics of the system in terms of slow motions of the aromatic cores that are determined by disentanglements of the alkoxy tails whose local motions are on a much shorter and faster scales. The presence of more than one configuration in the system (static disorder on the time scale of the QENS experiment) will lead to relatively long-range correlations in discotic liquid crystals with nonextensive statistics on the scale of the core diameter fast dynamics which influences dynamics on longer time scale exhibiting strong hierarchical structure. Such correlations have been taken into account by means of the number of subsystems that occupy the equilibrium state, providing a good picture of the complex dynamics of discotic liquid crystals and how these vary as a function of temperature and phase. It is likely that many other systems could be reanalyzed in the physical manner of this model that we have described here, which would lead to a better understanding of the evolution of underlying dynamics in terms of the physical parameters that are produced within model.
[1] F. M. Mulder, J. A. Stride, S. J. Picken, P. H. J. Kouwer, M. P. de Haas, L. D. A. Siebbeles, and G. J. Kearley, J. Am. Chem. Soc. 125, 3860 (2003).

[2] M. M. Elmahdy, G. Floudas, M. Mondeshki, H. W. Spiess, X. Dou, and K. Müllen, Phys. Rev. Lett. 100, 107801 (2008).
[3] D. Markovitsi, F. Rigaut, M. Mouallem, and J. Malthête, Chem. Phys. Lett. 135, 236 (1987).

[4] S. P. Brown, I. Schnell, J. D. Brand, K. Müllen, and H. W. Spiess, J. Am. Chem. Soc. 121, 6712 (1999).

[5] D. Andrienko, V. Marcon, and K. Kremer, J. Chem. Phys. 125, 
124902 (2006).

[6] A. Troisi, D. L. Cheung, and D. Andrienko, Phys. Rev. Lett. 102, 116602 (2009).

[7] J. Kirkpatrick, V. Marcon, K. Kremer, J. Nelson, and D. Andrienko, J. Chem. Phys. 129, 094506 (2008).

[8] C. W. Struijk, A. B. Sieval, J. E. J. Dakhorst, M. van Dijk, P. Kimkes, R. B. M. Koehorst, H. Donker, T. J. Schaafsma, S. J. Picken, A. M. van der Craats, J. M. Warman, H. Zuilhof, and E. J. R. Sudhölter, J. Am. Chem. Soc. 122, 11057 (2000).

[9] L. van Hove, Phys. Rev. 95, 249 (1954).

[10] G. R. Luckhurst and C. A. Veracini, in The Molecular Dynamics of Liquid Crystals, edited by G. R. Luckhurst (Kluwer Academics, Dordrecht, 1994), Vol. 1, Chap. 16, p. 451.

[11] W. K. Kegel and A. van Blaaderen, Science 287, 290 (2000).

[12] Alkoxy side chain deuteration was achieved via reaction of 2,3,6,7,10,11-hexa-hydroxy triphenylene with predeuterated $n$-hexyl bromide (Aldrich) under basic conditions $\left(\mathrm{K}_{2} \mathrm{CO}_{3}\right)$ in 2-butanone under reflux conditions for $24 \mathrm{~h}$ in a nitrogen atmosphere.

[13] A. Oleinikova, I. Brovchenko, A. Krukau, and A. K. Mazur, EPL 82, 46002 (2008).

[14] A. Ott, J. P. Bouchaud, D. Langevin, and W. Urbach, Phys. Rev. Lett. 65, 2201 (1990).

[15] P. F. Nealey, R. E. Cohen, and A. S. Argon, Polymer 36, 3687
(1995).

[16] T. Kanaya and K. Kaji, Adv. Polym. Sci. 154, 87 (2001).

[17] A. Arbe, J. Colmenero, F. Alvarez, M. Monkenbusch, D. Richter, B. Farago, and B. Frick, Phys. Rev. E 67, 051802 (2003).

[18] R. Metzler and J. Klafter, Phys. Rep. 339, 1 (2000).

[19] Yu. A. Berlin, A. L. Burin, and S. F. Fischer, Chem. Phys. 220, 25 (1997).

[20] C. Tsallis, Braz. J. Phys. 29, 1 (1999).

[21] A. N. Petridis, Cent. Eur. J. Phys. 4, 363 (2006).

[22] S. Chandrasekhar, Rev. Mod. Phys. 15, 1 (1943).

[23] C. T. Chudley and R. J. Elliott, Proc. Phys. Soc. London 77, 353 (1961).

[24] M. Bee, Quasielastic Neutron Scattering (Adam Hilger, Bristol, 1988).

[25] O. Kruglova, F. M. Mulder, A. Kotlewski, S. J. Picken, S. Parker, M. R. Johnson, and G. J. Kearley, Chem. Phys. 330, 360 (2006).

[26] S. V. Dvinskikh, I. Furó, H. Zimmermann, and A. Maliniak, Phys. Rev. E 65, 050702(R) (2002).

[27] G. Cinacchi, R. Colle, and A. Tani, J. Phys. Chem. B 108, 7969 (2004).

[28] S. Denisov, Phys. Lett. 235, 447 (1997).

[29] J. Klafter, A. Blumen, and M. F. Shlesinger, Phys. Rev. A 35 , 3081 (1987). 